\title{
Observaciones a la "instrucción el proyecto y la ejecución de las obras de hormigón pretensado" EP-77
}

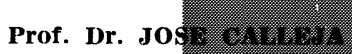

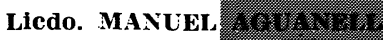
Cementos porthand VALDER: $4.40 .0 \%$

\section{R E S U M E N}

Se llama la atención sobre los límites máximos tolerados para el contenido de ion cloro en los cementos utilizables para hormigón pretensado, según las Instrucciones EP-77 y EP-80, para el proyecto y la ejecución de obras de dicho material.

Se hace destacar el hecho de que dichas Instrucciones sólo adoptan parte de unas Recomendacones Prácticas de la FIP y del CEB*; con exclusión de las limitaciones relativas al propio hormigón.

Por consiguiente, se pone de relieve la falta de correlación entre las limitaciones para el cemento y para el agua de amasado, en relación con la limitación aconsejada para el hormigón por las citadas Recomendaciones, en lo que al contenido máximo de ion cloro se refiere.

A base de razonamientos numéricos y de consideraciones de diversa índole, entre las que destacan la posibilidad de utilización de materiales hoy desechados, y de ahorro de energía en la molienda del cemento, se justifica y propone, previa comprobación experimental si es preciso, una elevación de los limites actuales del contenido de ion cloro de los cementos y de las aguas de amasado, según las mencionadas Instrucciones, a valores más altos, sin detrimento alguno para el hormigón y/o sus armaduras.

\section{ANTECEDENTES}

La "Instrucción" EP-77 (1) a la que hace referencia el título de este trabajo, fue aprobada por Real Decreto 1408/1977, de fecha 18 de febrero, y su texto publicado en el B.O.E. número 148, de fecha 22 de junio de 1977. Su vigencia comenzó a partir de los dos años de dicha publicación. Lleva, por lo tanto, algo más de un año en vigor.

* FIP = Fédération Internationale de la Précontrainte.

$\mathrm{CEB}=$ Comité Européen du Bèton. 
En-su Capítulo II: "Materiales" y en su Artículo 7.": "Cemento", se indica en el párrafo segúndo del Apartado 7.1.: "Cementos utilizables", que: "el contenido de ion cloro en el cemento, determinado con arreglo al método de ensayo descrito en el Anejo núm. 3 (de dicha Instrucción EP-77) deberá limitarse a un máximo de 0,03 en peso".

En Real Decreto 1780/1980, de fecha 14 de abril, por el que se modifica la EP-77, publicado en el B.O.E. núm. 216 de fecha 8 de septiembre de 1980, se dispone la aprobación de las modificaciones a dicha Instrucción, las cuales figuran en el Anexo al mencionado Decreto. Con tales modificaciones introducidas la EP-77 pasa a denominarse EP-80.

Las modificaciones de que se trata en lo que sigue se refieren, una, al citado párrafo segundo, en el que se señala que la limitación de referencia deberá ser de un máximo de 0,03 por ciento en peso; y, otra, al Apartado 9.1.: "Precisión" (de los resultados) del "Método de Ensayo para la Daterminación de Cloruros en Cemento y Aridos para la Construc. ción", incluido en el Anexo 3: "Métodos de Ensayo", de (1). Esta modificación puntualiza que "los resultados de dos ensayos no deberán diferir entre sí en más del 0,005 por" ciento" - la EP-77 indicaba 0,05 por ciento, lo cual chocaba con la limitación del 0,03 por ciento-.

De esta última modificación y, en general, de la validez — sensibilidad, precisión, fidelidad y reproducibilidad - del método que se da por admisible, no se va a tratar aquí.

Tan sólo se hace una pequeña observación relativa a la Nota 1 del texto del Método antes citado, cuyo contenido reza así en la EP-77: "Operando en las condiciones descritas en el presente método, es posible valorar con exactitud en estos materiales, contenidos de cloruros de hasta 0,20 por ciento. Este margen de apreciación puede elevarse al doble o al triple (0,4 ó 0,6 por ciento) partiendo de 0,5 gramos de muestra, en lugar de 1 gramo indicado en el método, y/o tomando ...". Se entiende que el texto quedaría más claro y preciso redactado así: "En las condiciones descritas a continuación, el método permite determinar con exactitud, en los materiales ensayados, contenidos de ion cloro inferiores a 0,20 por ciento. Este límite superior de aplicabilidad se puede elevar al doble o al triple (0,4 ó 0,6 por ciento) partiendo de 0,5 gramos de muestra, en lugar de 1 gramo como se indica en el método, y/o tomando ...".

Otra pequeña observación es la referente a que determinados ensayos llevados a cabo entre laboratorios en los últimos años, relativos a la determinación de componentes minoritarios en los cementos, han puesto de manifiesto que, dentro de las mayores dispersiones expresadas por los coeficientes de variación de las series de resultados estudiadas, figuran las correspondientes a las determinaciones de cloruros, para contenidos de éstos del orden de las centésimas por ciento. El coeficiente de variación en una serie de 35 resultados comprendidos entre $0,01 \%$ y $0,74 \%$, con una media de $0 ; 05 \%$, fue aproximadamente de 257 por ciento, sin eliminación de "resultados aberrantes"; y del orden del 43 por ciento con eliminación de 7 de dichos valores, situados todos en la cabeza de la serie. Hay que añadir que el número de tales resultados eliminados — siete- hubo de ser el máximo (exactamente $1 / 5$ del total de la serie), en comparación con los eliminables en las series de valores resultantes de las determinaciones de otros componentes minoritarios del cemento $(2)$.

Más recientemente, el coeficiente de variación ha sido, para otra serie de resultados análoga -37 valores comprendidos entre $0,01 \%$ y $0,18 \%$, con una media de $0,02 \%$, del orden de 170 por ciento - y de 0 por ciento, previa eliminación de 5 "resultados aberrantes" (aproximadamente $1 / 7$ de los totales de la serie) asimismo situados todos a la cabeza de la misma- (3). 
Estos últimos resultados, si bien hay que reconocer que suponen una mejora con el tiempo, distan mucho de ser alentadores y, sobre todo, fiables, ya que, en el mejor da los casos, las series contienen valores individuales "aberrantes" que son del orden de diez o quince veces superiores a las medias respectivas.

Por otra parte, en los resultados de las series comentadas se observa una tendencia clara a que los resultados aberrantes lo sean por exceso, con relación a las medias, dado que en ambas series la eliminación de los mismos es preciso hacerla exclusivamente por la cabeza, es decir, afectando sólo a los resultados más altos de las series.

Aparte de esto, se han estudiado los resultados de una combinación de métodos y variantes de los mismos, aplicados distinto número de veces a diferentes cantidades de muestra de partida - las más adecuadas en cada caso-, y a diez materiales de naturaleza distinta -entre ellos cemento- (4). El estudio ha permitido observar que alguno de los métodos turbidimétricos o nefelométricos, en alguna de sus variantes, da en los diez casos los valores mínimos minimorum para los contenidos porcentuales de ion cloro. Pero, al mismo tiempo, y en ocho de los diez casos, dan también los valores máximos maximorum - y en los otros dos casos, valores muy próximos a ellos-. Dicho de otro modo, a estos métodos corresponden las series más dispersas de valores.

En cuanto a las medias, alguno de los citados métodos o alguna de sus variantes dan en seis de los diez casos las más bajas, en tres de ellos las más altas, no distando mucho de serlo la del caso restante.

Se indica (4) que el método turbidimétrico en sus diversas modalidades da los valores más discordantes de todos los demás métodos, defecto que se considera corregible sólo en parte. Y se añade también que la repetibilidad del método es inferior a la de los otros métodos.

Por otro lado, se observa asimismo que, tanto los métodos turbidimétricos como los nefelométricos no se cuentan entre los de mayor precisión. En realidad es fácil observar que los coeficientes de variación de estos métodos son, según la variante de los mismos y la naturaleza del material al que se apliquen, del orden de 2 a 18 veces mayores que los de los demás métodos; y también que las relaciones entre los valores máximos y los mínimos son asimismo mayores que en el caso de otros métodos. Finalmente, al comparar los méto. dos turbidimétricos con los nefelométricos, se hace observar que la desviación standard de los resultados de los primeros es mayor que la correspondiente a los segundos.

Es evidente que estas circunstancias pueden resultar sistemáticamente perjudiciales para los cementos, al atribuirles a éstos valores del contenido de cloruros superiores a los reales, sobre todo cuando por los motivos que sean, se fijan limitaciones máximas tolerables para dichos contenidos.

En lo que sigue se va a tratar sólo de lo que se refiere a la limitación del contenido de cloruros -expresados como ion cloro $\mathrm{Cl}^{-}$- a un 0,03 por ciento en peso, en los cementos utilizables para hormigón pretensado. $\mathrm{Y}$ en esto conviene hacer un poco de historia, a] hilo de las siguientes consideraciones.

\section{CONSIDERACIONES}

Gran parte de las Normas, Instrucciones, Pliegos Generales, etc. españoles de todos los tiempos han sido y son transcripciones más o menos afortunadas de documentos extranje. ros análogos. Por documentos extranjeros análogos hay que entender también a estos efec- 
tos y en algunos casos - aunque desafortunadamente-, las simples recomendaciones (o incluso proyectos o propuestas de recomendaciones) que a uno u otro título más o menos justificado se han hecho y hacen allende nuestras fronteras. Recomendaciones que, como tales, o en estado de proyecto o propuesta, se adoptan a carga cerrada y sin discriminación. $\mathrm{y}$ a las que a veces se les confiere fuerza de ley.

Con ser esto malo, no es lo peor. Lo peor es transcribir tales recomendaciones sólo en parte - la que en cada caso y según los fines conviene-, con olvido o menosprecio del resto.

Las limitaciones de cloruros -ion cloro $\mathrm{Cl}^{-}$-, sulfatos $-\mathrm{SO}_{3}$ ó $\mathrm{SO}_{4}{ }^{2-}-$, y azufre en estado de sulfuro y/o de sulfito $-\mathrm{S}^{2-} \mathrm{y} / \mathrm{o} \mathrm{SO}_{2}$ ó $\mathrm{SO}_{3}{ }^{2-}$ - en cementos, aguas, áridos, aditivos y hasta en los propios hormigones en conjunto, ha sido durante tiempo uno de los caballos de batalla de la FIP, del CEB, de la RILEM* y de otros varios organismos internacionales similares. Así, por ejemplo, y por citar una referencia de última hora, la Asociación Científica del Pretensado ha publicado recientemente unas Informaciones (5) en las que, entre otras cosas, se da cuenta de la actividad de la Comisión Internacional de Du. rabilidad de las Construcciones Pretensadas, de la FIP, presidida por M. F. DUMAS, a lo largo de sus reuniones desde 1967 en París, hasta el 6. ${ }^{\circ}$ Congreso de la FIP en Praga y junio de 1970.

En estas Informaciones se indica que los objetivos de la mencionada Comisión fueron dos: uno, inventariar las obras en pretensado desde el punto de vista de la durabilidad; y, otro, fijar "recomendaciones" para evitar los incidentes o accidentes que fueren puestos de manifiesto por el inventario o encuesta previos.

En cuanto al segundo objetivo las recomendaciones afectaron a los elementos constitutivos del hormigón pretensado en general y, de forma particular, a los del componente pétreo del material: áridos, cemento, aguas de amasado y aditivos, así como al conjunto, es decir, al hormigón en sí.

Por lo que se refiere a los áridos, éstos no fueron hasta el momento objeto de recomendaciones precisas, plasmadas en limitaciones cuantitativas de los contenidos de elementos 0 compuestos nocivos - salvo en lo que afecta al contenido de sulfatos, limitado a 1,2 kilogramos por metro cúbico de hormigón-. Las limitaciones cuantitativas de los contenidos de cloruros y sulfuros en los áridos quedaron pendientes de estudio y aplazadas, ... "ad calendas graecas". La Comisión se contentó con recomendar que dichos contenidos fueran "lo más reducidos posible" (5), lo cual ni siquiera consta así en las Recomendaciones CEB-FIP (6) emanadas del 6." Congreso de la FIP, de 1970 en Praga.

En cambio, las recomendaciones de ta Comisión FIP de Durabilidad (5) proponen para los cementos, las aguas de amasado y los propios hormigones, las limitaciones siguientes:

Para los cementos:

$$
\begin{array}{ll}
\mathrm{Cl}^{-} & 0,03 \% \text { máximo } \\
\mathrm{S}\left(\mathrm{S}^{2-} \text { ó } \mathrm{SO}_{3}{ }^{2-}\right) & 0,20 \% \text { máximo } \\
\mathrm{SO}_{3} & 3,50 \% \text { máximo** }
\end{array}
$$

* RILEM :- Réunion Internationale de Laboratoires d'Essais —et de Recherches- sur les Matériaux.

* Para ambientes no agresivos por fuertes cantidades de sulfatos. Hasta $5 \%$ en cementos siderúrgicos - de escorias_con un contenido igual o menor de $20 \%$ de clínker e igual o mayor de $80 \%$ de escoria. Para ambientes fuertemente agresivos por sulfatos, cementos especiales resistentes a aguas selenitosas $y$ de mar, con bajo o nulo contenido de C:A. 
Para las aguas de amasado:

$$
\begin{array}{ll}
\mathrm{Cl}^{-} & 0,02 \% \text { máximo } \\
\mathrm{SO}_{3} & 0,10 \% \text { máximo }
\end{array}
$$

Para los hormigones:

$$
\begin{array}{ll}
\mathrm{Cl}^{-} & 0,05 \% \text { máximo } \\
\mathrm{SO}_{3} & 0,64 \% \text { máximo }
\end{array}
$$

La EP-77 (y la actual EP-80) establecen, entre otras, las limitaciones siguientes (traducidas a expresiones FIP-CEB):

Para los cementos*:

$$
\mathrm{Cl}^{-} \quad 0,03 \% \text { máximo }
$$

Para las aguas de amasado:

$$
\begin{array}{ll}
\mathrm{Cl}^{-} & 0,025 \% \text { máximo } \\
\mathrm{SO}_{3} & \sim 0,085 \% \text { máximo }
\end{array}
$$

Para los áridos:

$$
\begin{aligned}
& \mathrm{Cl}^{-} \quad 0,03 \% \text { máximo (fino y grueso) } \\
& \mathrm{SO}_{3} \quad \sim 0,40 \% \text { máximo (fino) } \\
& 1,00 \% \text { máximo (grueso) }
\end{aligned}
$$

Por el contrario, no establecen ninguna limitación relativa a los hormigones en conjunto.

Parece lógico pensar que si un componente químico en unas proporciones dadas puede ser nocivo para el hormigón por unas razones cualesquiera, lo sea por el hecho de encontrarse en el hormigón, con independencia de cuál sea su origen, es decir, de cuál o cuáles sean los materiales del hormigón que lo aporten en una u otra medida.

Esto es algo generalmente aceptado** y, como ejemplo adicional, sirva y baste el hecho —comprobado (7) (8) - de que en la reacción expansiva árido-álcalis no sólo cuentan los álcalis aportados por el cemento, sino la totalidad de los álcalis presentes en el hormigón, vengan de donde vinieren. Con lo cual resulta que, como ha sido demostrado hasta la saciedad, con unos áridos susceptibles a los álcalis - no del cemento, sino en general-, pueden tener lugar reacciones expansivas con cementos de bajo contenido de álcalis -menos de 0,6 por ciento de $\mathrm{Na}_{2} \mathrm{O}$ equivalente $\left(\mathrm{Na}_{2} \mathrm{O} \%+0,658 \mathrm{~K}_{2} \mathrm{O} \%\right)$ - y no tenerlo con cementos de contenido de álcalis mucho mayor. Todo ello claro está, en función del aporte de álcalis por parte de otros materiales y, en definitiva, de que el contenido total de álcalis del hormigón esté por encima o por debajo de un determinado umbral, con independencia del contenido de álcalis del cemento.

* Obsérvese que no se limita el contenido de azufre en forma de sulfuros. A mayor abundamiento, no se descarta el empleo de cementos siderúrgicos para hormigón pretensado, pudiéndose utilizar éstos mediante una justificación especial.

* Las propias recomendaciones de la Comisión FIP de Durabilidad condicionan el contenido de sulfatos de los áridos a que en el hormigón no pasen de 1,2 kilogramos por metro cúbico, como queda indicado antes 
Mutatis mutandis, lo mismo vale para el contenido de ion cloro en el hormigón pretensado: lo que cuenta es el contenido total de dicho ion, al margen del origen del mismo, e independientemente del contenido de ion cloro en el cemento.

A este respecto, vale la pena fijarse en la limitación al 0,05 por ciento máximo que se hace en las "Recomendaciones" citadas (5), y en la incidencia que tal limitación tiene en unos posibles topes para el contenido de ion cloro en el cemento y en el agua de amasado, supuesto que se ignoren los áridos a tal efecto, como de hecho sucede en (5) y (6).

Imagínese, con arreglo al Cuadro 1, una serie de hormigones con diferentes dosificaciones de cemento —entre 250 y $450 \mathrm{~kg} / \mathrm{m}^{3}$ - y distintas relaciones agua/cemento -entre 0,5 y 0,7-, por abarcar un amplio margen, con independencia de la mayor o menor verosimilitud de algunos de dichos hormigones en la realidad. En todo caso, muchos de ellos son verosímiles y la serie abarca prácticamente todas las posibilidades: de alta dosificación y alta o baja relación agua/cemento; de baja dosificación y baja o alta relación agua/cemento, y casos intermedios.

Imagínese también - por simplificar y fijar ideas - que los hormigones del Cuadro 1 son de 2.400 kilogramos por metro cúbico. Con tal masa y un contenido máximo admisible de 0,05 por ciento - en peso- de ion cloro, tales hormigones podrían contener, como máximo, 1,2 kilogramos de ion cloro por metro cúbico. Repartido este contenido' entre el cemento y el agua, proporcionalmente a los pesos de ambos componentes -áridos aparte-, las cantidades proporcionales de ion cloro en uno y otro, para los veinticinco casos del Cuadro 1 serían las indicadas en el Cuadro 2 . Y expresadas en los respectivos tantos por ciento relativos al cemento y al agua de amasado, serían las indicadas en el Cuadro 3.

El cuadro 3 pone de relieve que para un hormigón cualquiera de los contemplados en los casos del Cuadro 1 tenga un contenido máximo de ion cloro de 0,05 por ciento en peso, basta y sobra con que, tanto el agua de amasado como el cemento - aparte los áridostengan contenidos máximos de dicho ion comprendidos entre un límite inferior de 0,16 por ciento y un límite superior de 0,32 por ciento - en números redondos, entre $0,15 \mathrm{y}$ 0,30 por ciento-.

\section{CUADRO 1}

Agua de amasado de hormigones: $l / m^{3}$

\begin{tabular}{|c|c|c|c|c|c|c|c|c|c|c|}
\hline \multirow[b]{2}{*}{ Relación a/c } & \multicolumn{10}{|c|}{ Dosificación de cemento: $\mathrm{kg} / \mathrm{m}^{3}$} \\
\hline & \multicolumn{2}{|c|}{250} & \multicolumn{2}{|c|}{300} & \multicolumn{2}{|c|}{350} & \multicolumn{2}{|c|}{400} & \multicolumn{2}{|r|}{450} \\
\hline 0,50 & & 125 & 6) & 150 & 11) & 175 & 16) 2 & 200 & 21) & 225 \\
\hline 0,55 & & 137,5 & 7) & 165 & 12) & 192,5 & 17) 2 & 220 & 22) & 247,5 \\
\hline 0,60 & 3) & 150 & 8) & 180 & 13) & 210 & 18) 2 & 240 & 23) & 270 \\
\hline 0,65 & & 162,5 & 9) & 195 & 14) & 227,5 & 19) 2 & 260 & 24) & 292,5 \\
\hline 0,70 & & 175 & 10) & 210 & 15) & 245 & 20) 2 & 280 & 25) & 315 \\
\hline
\end{tabular}




\begin{tabular}{|c|c|}
\hline | & $\begin{array}{l}\text { Casos del } \\
\text { cuadro } 1\end{array}$ \\
\hline 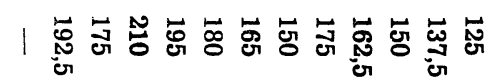 & Agua $\mathrm{kg}$ \\
\hline | & $\begin{array}{l}\mathrm{Cl}^{-} \text {en el } \\
\mathrm{Agua} \\
\mathrm{kg}\end{array}$ \\
\hline | & $\begin{array}{l}\text { Cl- en el } \\
\text { Agua \% }\end{array}$ \\
\hline | 岁 㟧 & Cemento $\mathrm{kg}$ \\
\hline | & $\begin{array}{l}\text { Cl- en el } \\
\text { Cemento } \mathrm{kg}\end{array}$ \\
\hline | & $\begin{array}{l}\text { Cl- en el } \\
\text { cemento } \%\end{array}$ \\
\hline 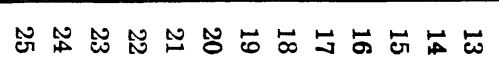 & $\begin{array}{l}\text { Casos del } \\
\text { cuadro } 1\end{array}$ \\
\hline 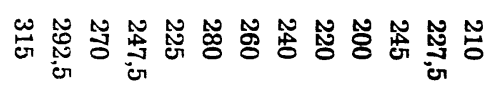 & Agua $\mathrm{kg}$ \\
\hline 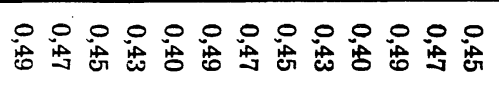 & $\begin{array}{l}\text { Cl-en el } \\
\text { Agua } \mathrm{kg}\end{array}$ \\
\hline 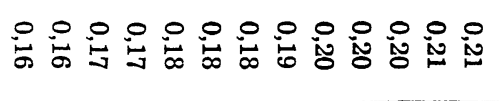 & $\begin{array}{l}\text { Cl-en el } \\
\text { Agua \% }\end{array}$ \\
\hline 岁 岁 & Cemento $\mathrm{kg}$ \\
\hline 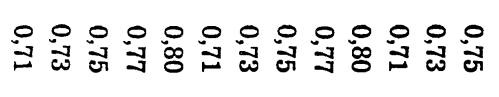 & $\begin{array}{c}\text { Cl- en el } \\
\text { cemento } \mathrm{kg}\end{array}$ \\
\hline 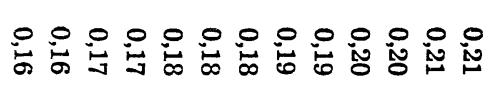 & $\begin{array}{l}\text { Cl- en el } \\
\text { cemento } \%\end{array}$ \\
\hline
\end{tabular}

(c) Consejo Superior de Investigaciones Científicas Licencia Creative Commons 3.0 España (by-nc)

\begin{tabular}{|c|c|}
\hline | $\approx \Xi 50 \infty \sim \infty$ O & $\begin{array}{l}\text { Casos del } \\
\text { cuadro } 1\end{array}$ \\
\hline 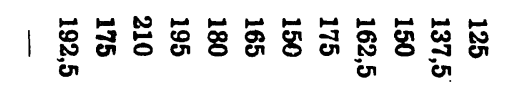 & Agua $A \mathrm{~kg}$ \\
\hline 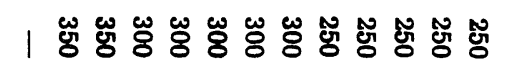 & Cemento $\mathrm{C}$ kg \\
\hline 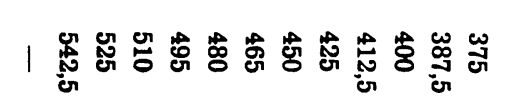 & $\underset{\text { Agua } y}{\text { cemento } A+C^{-}}$ \\
\hline 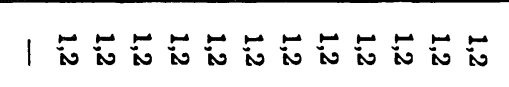 & $\begin{array}{l}\mathrm{Cl}^{-} \mathrm{en} \\
\mathrm{A}+\mathrm{C} \mathrm{kg}\end{array}$ \\
\hline 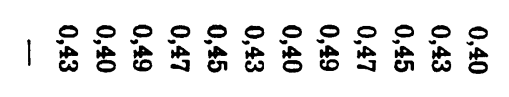 & $\mathrm{Cl}^{-}$en $\mathbf{A} \mathbf{~ k g}$ \\
\hline | & $\mathrm{Cl}-$ en $\mathbf{C} \mathrm{kg}$ \\
\hline 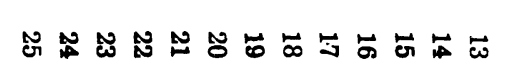 & $\begin{array}{l}\text { Casos del } \\
\text { cuadro } 1\end{array}$ \\
\hline 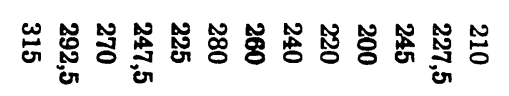 & Agua $\mathrm{A} \mathrm{kg}$ \\
\hline 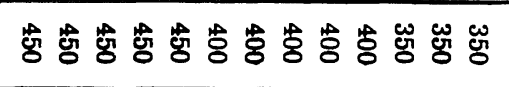 & Cemento $\mathrm{C} \mathrm{kg}$ \\
\hline 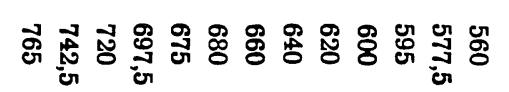 & $\begin{array}{l}\text { Agua } y \\
\text { cemento } \mathrm{A}+\mathrm{C}\end{array}$ \\
\hline 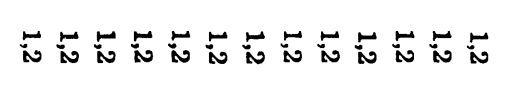 & $\begin{array}{l}\stackrel{C l-e n}{\text { en }} \\
\mathrm{A}+\mathrm{C} \mathrm{kg}\end{array}$ \\
\hline 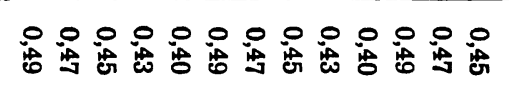 & $\mathrm{Cl}^{-}$en $\mathrm{A} \mathbf{~ k g}$ \\
\hline 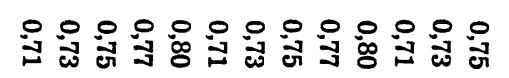 & $\mathrm{Cl}^{-}$en $\mathbf{c} \mathbf{~ k g}$ \\
\hline
\end{tabular}

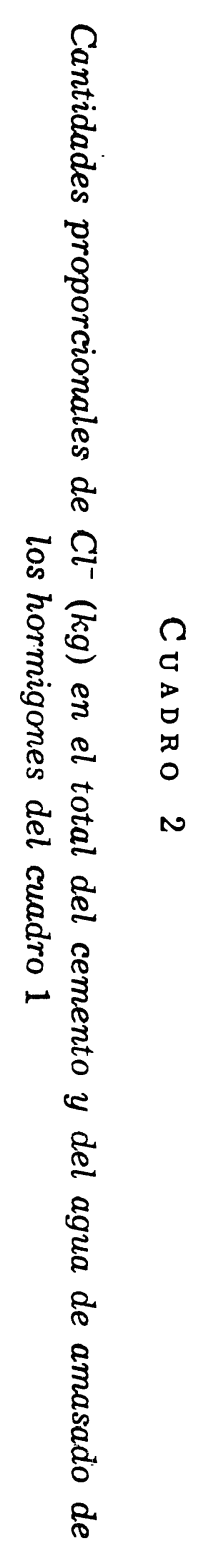


Es claro que estos valores porcentuales de ion cloro son, en cada caso 5 y 10 veces superiores al valor máximo tolerable fijado por las Recomendaciones (5) y por las EP-77 y EP-80 para el cemento $(0,03 \%)$, y 7,5 y 15 veces -ó 6 y 12 veces, respectivamente-, superiores al valor máximo tolerable fijado por dichas recomendaciones e instrucciones para el agua de amasado.

Si se considera el caso más desfavorable -0 , si se prefiere, el situado más del lado de la seguridad-, habrá que tener en cuenta el valor mínimo minimorum de dicha gama de valores, es decir, el de 0,15 por ciento, el cual es, en todo caso y muy aproximadamente, cinco veces superior al límite máximo de tolerancia fijado por las Instrucciones EP-77 y EP-80 para el contenido de ion cloro, tanto en los cementos como en las aguas de amasado para el hormigón pretensado.

Por otra parte, habida cuenta de que el agua de amasado interviene en el hormigón en una proporción menor que la del cemento, parecería lógico que el límite de tolerancia para el contenido de ion cloro en el agua fuese proporcionalmente más elevado que el del cemento. Es decir, que para un hormigón con una relación agua/cemento intermedia de 0,6 -entre 0,5 y 0,7 del Cuadro 1-, si el límite para el cemento fuese de $x \%$, el del agua de amasado debería ser del orden de $1 / 0,6 x \%$, esto es, de $1,67 x \%$. Por ejemplo, al límite de $0,03 \%$ para el cemento debería corresponder un límite de $0,03.1,67=0,05 \%$ para el agua, y, sin embargo, el límite contemplado para ésta en las EP-77 y 80 es justamente la mitad: $0,025 \%$ y más bajo aún en las Recomendaciones (5): $0,020 \%$.

Otra cuestión es la relativa a la actividad del cloro a efectos corrosivos de las armaduras. Es activo - despasivante del acero- el cloro iónico móvil de los cloruros, pero no lo es el cloro combinado e inmovilizado de forma compleja con los constituyentes del cemento hidratado, y particularmente con los aluminatos, para formar el conocido cloroaluminato cálcico complejo o "sal de FRIEDEL": $\mathrm{C}_{3} \mathrm{~A} \cdot \mathrm{CaCl}_{2} \cdot 10 \mathrm{H}_{2} \mathrm{O}$, que es el compuesto que se forma con el cloruro cálcico que se añade a veces al hormigón - no pretensado-, como acelerador del fraguado y del endurecimiento.

Quiere esto decir que, al menos una parte del ion cloro de los cloruros contenidos en el cemento y/o en el agua de amasado -y circunstancialmente en los áridos-, se fija e inmoviliza en forma de cloroaluminato, con lo cual queda incapacitado para producir efectos corrosivos, a no ser que se movilice por descomposición de dicho compuesto, por ejemplo bajo la acción de algún ataque al hormigón por algún agresivo externo. Pero esta posibilidad no se da en todos los hormigones pretensados, ni en los casos en que se pueda dar su probabilidad es grande. Precisamente el hormigón pretensado es esencialmente un material muy cuidado en cuanto a su cálculo, componentes, proporciones y elaboración.

Otro aspecto digno de comentario es la reticencia de las tan citadas "Recomendaciones" a especificar sobre contenidos máximos admisibles de ion cloro en los áridos. ¿Por qué?.

Una explicación podría ser el escaso significado que dichos contenidos tendrían, en general, en el tema tratado. En efecto, centrando la atención en los áridos gruesos, éstos no tienen el claro -en el caso de tenerlo- "a flor de piel", es decir, en la superficie, de forma que al contacto de aquéllos con el agua de amasado se convierta en ion cloro. Incluso es dudoso - según los casos- que dicho cloro o todo él se encuentre en forma directamente ionizable. Pero, en cualquier caso, ha'y que pensar que el cloro se halla bastante homogénea y uniformemente repartido en toda la masa del árido y de sus individuos. En estas condiciones, y dada la masa, el tamaño, la compacidad y la impermeabilidad de los áridos normales empleados en el hormigón pretensado, ¿cómo pensar que dicho cloro - aún admitiendo que se encontrase en su totalidad en forma directamente ionizable - iba a salir de la masa del árido para pasar al agua de amasado? Algo muy distinto es pensar 
en la utilización como árido -en otros casos distintos del pretensado, claro está- de arenas de playa sin lavar, las cuales sí contienen cloruros y muchos, y casi exclusivamente "a flor de piel".

Pero, eso sí, para determinar el contenido de cloro de un árido - sea cualquiera - es preciso molerlo a un tamaño menor de 200 micias, haciéndolo pasar por un tamiz de 900 mallas $/ \mathrm{cm}^{2}$, y atacarlo con ácido nítrico - Anejo 3 de la referencia (1)_, con lo cual todo el cloro del árido pasa a la forma iónica, aun cuando inicialmente no se encontrase en su totalidad en forma ionizable. Con esto y con las actuales limitaciones de (1) es posible rechazar áridos que podrían ser perfectamente utilizables; de la misma manera es posible rechazar cementos sin justificación alguna, o al menos suficiente.

El caso del cemento merece otra consideración. Sabido es el interés general que existe en la actualidad por el ahorro de energía en los procesos industriales de fabricación, y particularmente en el del cemento. Interés que está llevando a usar nuevamente carbones en la clinkerización, a emplear adiciones activaš, así como a otras medidas encaminadas a dicho fin.

Una de estas medidas puestas en práctica en todo tiempo y lugar ha sido y es la de utilizar coadyuvantes de molienda para ahorrar kilowatios en la molturación del clínker, sin menoscabo de la finura del cemento. Estos coadyuvantes son de distintos tipos (productos orgánicos tensoactivos de naturaleza alcohólica, amínica, ácida, sulfónica o mixta aún más compleja), entre los cuales destaca por su conocimiento y vasto empleo la trietanolamina. Pues bien, algunos de los productos más recientes con estas características contienen cloro, el cual queda retenido, al menos en parte, en la superficie del cemento. Sin embargo, es tan baja la proporción en que es preciso utilizar estos coadyuvantes, que tal retención es mínima y absolutamente desdeñable. Pero tal vez no tanto como para que, en algún caso, no pueda invalidar a algún cemento para su empleo en hormigón pretensado, dada la injustificadamente rigurosa limitación del contenido de ion cloro en los cementos, según la Instrucción EP-80 vigente.

Con esto se "castiga" una vez más a los cementos, al dificultar el ahorro de energía en su fabricación, precisamente en unos tipos y clases tales que, como los cementos P-ARI del Pliego RC-75 (9), por su propiedad adicional de altas resistencias iniciales $-\mathrm{y}$ a toda edadrequieren -entre otras cosas- una molienda más fina y son, precisamente, los más idóneos para el pretensado y para determinar dos tipos de prefabricación.

\section{CONCLUSIONES}

3.1. Resulta extraño, y en cualquier caso parece insuficientemente justificado, adoptar de otros países u organismos internacionales, como normas oficiales de obligado cumplimiento, lo que en dichos países y organismos no pasan de ser meras "recomendaciones" - a veces sólo propuestaś o proyectos de recomendaciones- y, por añadidura incompletas.

3.2. Resulta aún más extraño que dicha adopción se haga con análoga injustificación, sólo de una parte, y no de la totalidad de dichas "recomendaciones" ya de por sí incompletas.

3.3. De adoptarlas en su totalidad resultaría, para el caso aquí tratado, que, tanto en las "recomendaciones" de referencia (5) como en las Instrucciones españolas EP-77 y EP-80 (1) que en parte las copian —o con ellas coinciden-, los límites máximos de tolerancia fijados para el contenido de ion cloro en los cementos utilizables para hormigón pretensado, así como en las aguas de amasado de los mismos, podrían ser. 
por lo menos, del orden de cinco veces mayores de lo que son en la actualidad, sin detrimento alguno para los hormigones con ellos confeccionados ni para sus armaduras.

3.4. Por contra, la elevación de dichos límites en la medida señalada posibilitaría la utilización, para hormigón pretensado, de cementos y aguas de amasado hoy por hoy injustificadamente rechazados.

3.5. Al propio tiempo, la elevación en cuestión ayudaría al ahorro de energía en la molienda de los cementos de altas resistencias iniciales P-ARI del Pliego RC-75, especialmente aptos para el pretensado, al hacer posible la utilización de coadyuvantes de molienda de naturaleza clorada.

3.6. En todo caso, no se puede negar el interés y la utilidad de realizar pruebas experimentales suficientes para poner en evidencia de manera inequívoca, la procedencia o no de elevar los límites señalados, en la medida indicada. De otro modo, determinados cementos, perfectamente aptos para el hormigón pretensado, permanecerán indefinidamente excluidos y "castigados".

\section{R E F E R E N C I A S}

(1) EP-77: Instrucción para el proyecto y la ejecución de obras de hormigón pretensado. Servicio de Publicaciones de la Secretaría General Técnica, del Ministerio de Obras Públicas, Madrid, 1977.

(2) Bombled, P. J. y Barrot, H.: “Essais Interlaboratoires (1978-1979)”. CERILH, París, julio 1979.

(3) Barrot, H. y LeVeque, R.: “Essais Interlaboratoires (1979-1980).” CERILH, Paris, julio 1980.

(4) Moreno Arus, F. y Esteve Sala, J.: "Determinación de cloruro en materias primas y productos terminados de la industria del cemento". Materiales de Construcción (IETCC), núm. 170, págs. 5-35 (1978).

(5) Informations A.S.P. (Association Scientifique de la Précontrainte), págs. 268-276, Lille, Mars-Avril 198u.

(6) Recommandations Pratiques FIP-CEB, Propositions (1.ere édition) et Principes et Recommandations (2. 'me édition), 6 Congrès FIP de Prague, 1970.

(7) Veronelli, D. J. E.: “Durabilidad de los Hormıgones: Reacción Arido-Alcalis”. Materiales de Construcción (IETCC), núm. 171, págs. 5-33 (1978).

(8) Calleja, J.: "Durability”. Principal Report, Tema 7-II, 7." Congreso Internacional de la Química de! Cemento, París, julio 1980.

19) RC-75: Pliego de prescripciones técnicas generales para la recepción de cementos. Servicio de Publicaciones de la Secretaria General Técnica, del iMiisterio de Obras Públicas, Madrid, 1975. 